Martin Seeliger

\title{
Warum die EU aus gewerkschaftlicher Sicht keine Solidargemeinschaft darstellt
}

\section{Einleitung}

Unter Bedingungen des gemeinsamen Marktes und der Währungsunion hat die Europäische Union im Verlauf der letzten Jahre eine in vielerlei Hinsicht krisenhafte Entwicklung genommen. ${ }^{1}$ Angesichts von Austeritätspolitik, Flüchtlingskrise, Arbeitskräftemobilität und anhaltender Liberalisierungsbestrebungen durch den Europäischen Gerichtshof überträgt sich diese Dynamik heute mehr denn je in einen ergebnisoffenen Prozess der »Politisierung der EU «. ${ }^{2}$

Die Frage, wie dieser Krise konstruktiv zu begegnen sei, ist nicht zuletzt im Rahmen dieser Zeitschrift Gegenstand einer lebhaften Debatte gewesen. Während etwa Brunkhorst $^{3}$ zur Lösung der Konflikte die Stärkung der supranationalen Kompetenzen zwischen den Mitgliedstaaten als unumgänglich erscheint - den europäischen Gewerkschaften empfiehlt $\mathrm{er}^{4}$ eine "Transnationalisierung des demokratischen Klassenkampfes « -, weist Höpner ${ }^{5}$ entsprechende Vorschläge der Verlagerung nationaler Handlungspotenziale auf die EU-Ebene als »Integrationismus « zurück: Anstatt sich in wenig aussichtsreichen Internationalisierungsbestrebungen zu versteigen, sollten Gewerkschaften sich, wo diese bestehen, auf die Erhaltung nationaler Standards konzentrieren.

Im erstgenannten Sinne verwiesen zuletzt Loh und Skupien ${ }^{6}$ auf die tragende Rolle intermediärer Organisationen für die Entwicklung der Union zu einem krisenfesten Gemeinwesen. In Auseinandersetzung mit den Voraussetzungen und Konsequenzen der aktuellen Krise der EU gelangen die Autoren zu der These, "dass eine starke transnationale Gewerkschaftsbewegung und eine europäische Arbeitslosenversicherung die negativen Auswirkungen der bisher forcierten ökonomischen Integration ausbalancieren können ${ }^{7}{ }^{7}$ Ihren konzeptionellen Überlegungen zur Frage, welche Formen der Solidarität Gewerkschaften leisten könnten, um ein solches Gemeinwesen perspektivisch zu etablieren, legen die Autoren jedoch kein empirisches Verständnis der realen Praktiken internationaler gewerkschaftlicher Zusammenarbeit

1 Für wertvolle Anregungen bedanke ich mich bei Martin Höpner, Johannes Kiess sowie zwei anonymen Gutachter(inne)n.

2 Zürn 2013, S. 413.

3 Brunkhorst 2014 a.

4 Brunkhorst 2014 b, S. 166.

5 Höpner 2015.

6 Loh, Skupien 2016.

7 Ebd., S. 589. 
in der EU zugrunde. Die entscheidende Frage muss aber nicht lauten, ob Solidarität in der Kooperation denkbar ist, sondern ob sie praktiziert wird.

Den Faden dieser Diskussion greift der vorliegende Beitrag auf, um sie anschlieBend auf eine Reihe von Befunden aus einem Projekt zu gewerkschaftlicher Positionsbildung im Rahmen der EU zu beziehen, nämlich in den drei wichtigsten Feldern der europäischen Tarifpolitik, namentlich den Auseinandersetzungen um die Dienstleistungsfreiheit im gemeinsamen Markt, den europäischen Mindestlohn sowie die Initiativen zur Lohnkoordinierung im Euro-Raum. Die Frage, so die anzustellende Grundüberlegung, ob Gewerkschaften bedeutsame Akteure in einem Prozess der graduellen Stärkung der sozialen Dimension der EU sind, lässt sich vor allem mit Blick auf ihre Bedeutung im Bereich ihres Kerngeschäfts beantworten - der Tarifpolitik. Gelingt es den Gewerkschaften, hier gemeinsame Positionen zu vertreten und umzusetzen, spricht dies für eine grundsätzliche Möglichkeit der Etablierung signifikanter Gewerkschaftsmacht auf europäischer Ebene. Gelingt dies jedoch nicht, steht auch die Aussicht auf ein »Europäisches Sozialmodell « als den gemeinsamen Markt ergänzende Dimension infrage.

\section{Europäische Tarifpolitik in der Krise - Gibt es Grund zum Optimismus?}

Ausgangspunkt aktueller Krisendiagnosen ist die Feststellung, dass sich die europäische Integration seit den 1980er Jahren immer stärker über die Etablierung einer gemeinsamen Ökonomie vollzieht. Die vier Grundfreiheiten des europäischen Binnenmarktes (freier Warenverkehr, Personenfreizügigkeit, Dienstleistungsfreiheit und freier Kapital- und Zahlungsverkehr) und die gemeinsame Währung dienen wenigstens ihrer Idee nach - der engeren Zusammenarbeit wirtschaftlicher Akteure aus den einzelnen Mitgliedstaaten, während die Etablierung EU-weiter Normen zur Bewahrung nationaler Mindeststandards in der sozialpolitischen Gesetzgebung weitgehend bedeutungslos geblieben ist. ${ }^{8}$ Aus Sicht der Gewerkschaften ergibt sich hieraus die Gefahr einer systematischen Abwärtskonkurrenz nationaler Beschäftigungsregime im gemeinsamen Markt.

Durch die Erweiterungsrunden der Jahre 2004 und 2007 erfuhr die Europäische Union außerdem nicht nur eine Vertiefung ihrer politischen Kompetenzen, sondern auch eine Vergrößerung ihres Umfangs. Die hieraus resultierende politökonomische Heterogenität kann heute mehr denn je als charakteristisches Merkmal gelten, welches bereits mit dem Europamotto "Einheit in Vielfalt " zum Ausdruck gebracht wurde. Aus Sicht politischer Akteure, die - wie die europäischen Gewerkschaften gemeinsame (politische) Positionen im europäischen Rahmen zu etablieren versuchen, ergibt sich so eine Konstellation, in der »verschiedene Länder und spezielle Gruppen von Akteuren in diesen Ländern vor unterschiedlichen Problemen stehen und verschiedene Interessen, Traditionen und Ansichten darüber haben, welche Lösungen wünschenswert oder machbar sind.$^{9}{ }^{9}$

8 Scharpf 2012.

9 Keune 2008, S. 297. 
Die Frage, inwiefern es Gewerkschaften im Prozess der europäischen Integration gelingt, gemeinsame politische Positionen auf europäischer Ebene zu entwickeln, ist in der sozialwissenschaftlichen Auseinandersetzung von verschiedenen Blickpunkten aus beantwortet worden. Im Sinne von Keune ${ }^{10}$ lässt sich hier zwischen eurooptimistischen und europessimistischen Ansätzen unterscheiden. Während letztere vor allem den Tatbestand politökonomischer Heterogenität betonen, ${ }^{11}$ um die Probleme der Abstimmung gemeinsamer politischer Ziele sichtbar zu machen, erkennen optimistische Vertreter wie Brunkhorst ${ }^{12}$ eine unter den europäischen Lohnabhängigen »transnationale Klassenlage «, die durch die richtige Form der Auseinandersetzung nationaler Gewerkschaftsvertreter miteinander in ein gemeinsames "Klassenbewusstsein umschlagen « könne.

Für das (bisherige und/oder zukünftig wahrscheinliche) Ausbleiben einer effektiven transnationalen Organisation gewerkschaftspolitischer Abstimmung in der EU werden in der Literatur verschiedene Ursachen benannt. ${ }^{13}$ Zum einen seien Gewerkschaften als Organisationen unter spezifischen nationalstaatlichen Bedingungen (Wohlstandsniveau, Modi der Lohnfindung, Sättigung lokaler Arbeitsmärkte etc.) gewachsen, deren Ausprägung auch ihre Interessenlagen prägen. Auf nationaler Ebene übertrage sich dies, zweitens, in unterschiedliche Modelle der Vertretungspolitik, nicht nur hinsichtlich von Struktur und Einfluss der Organisationen, sondern auch mit Blick auf ihre politisch-ideologische Orientierung. ${ }^{14}$ Weitere Hindernisse für eine effektive Abstimmung nationaler politischer Positionen ergäben sich, drittens, vor unterschiedlichen kulturellen Hintergründen der jeweiligen nationalen Vertreter. Mit Blick auf die europäische Ebene bemerkt Bernaciak ${ }^{15}$ die »begrenzte Kompetenz der supranationalen EU Institutionen in der sozialen Sphäre «. Für den Bereich des europäischen Sozialdialogs korrespondiert diese allgemeine Schwäche der EU in der sozialpolitischen Regelsetzung außerdem mit der absichtsvollen Zurückhaltung der Kapitalseite. ${ }^{16}$

Indizien, die gegen die Etablierung effektiver gewerkschaftlicher Vertretung im politischen System der Europäischen Union sprechen, lassen sich also innerhalb sowie im Umfeld der nationalen und europäischen Gewerkschaftsorganisationen erkennen. Pessimistischen Argumenten folgend, klafft im Feld europäischer Gewerkschaftspolitik also eine Lücke zwischen Anspruch und Wirklichkeit. Deshalb, so der Tenor, bestehe unter den Regierungen der Mitgliedsländer »keine Aussicht mehr, sich auf mehr als minimale soziale Standards zu einigen ${ }_{.}{ }^{17}$

10 Keune 2012.

11 Vgl. Höpner, Schäfer 2012.

12 Brunkhorst 2014 a, S. 167.

13 Bernaciak 2010, S. 122; Ramsay 1999, S. 192; Platzer 2010; Hoffmann 2010, S. 100.

14 Hyman 2001.

15 Bernaciak 2010, S. 122.

16 Schäfer, Streeck 2008.

17 Höpner, Schäfer 2008, S. 28. 
Vergleicht man die pessimistische Position mit der Haltung optimistischer Beobachter, fällt auf, dass Diskrepanzen in der Wahrnehmung zwischen den beiden Lagern wohl nicht zuletzt vom jeweiligen Erkenntnisinteresse abhängen. Anliegen eurooptimistischer Beiträge ist es hierbei, die Möglichkeit und Notwendigkeit einer Europäisierung der Gewerkschaftspolitik zu begründen. ${ }^{18}$ In diesem Sinne stellt auch der Artikel von Loh und Skupien einen eurooptimistischen Beitrag dar.

In ihrem Text zur »EU als Solidargemeinschaft « stellen Wolf Loh und Stefan Skupien die »Frage nach der Integrationsfähigkeit und -möglichkeit der EU « ${ }^{19}$ mit Blick auf eine mögliche konstruktive Rolle der europäischen Gewerkschaften. Im Sinne des wichtigsten Leitwerts der Arbeiterbewegung, ${ }^{20}$ aber auch unter kohäsionstheoretischen Gesichtspunkten ${ }^{21}$ unterscheiden die beiden Autoren drei Formen von Solidarität in Bezug darauf, welche europäischen Gewerkschaften zur Stärkung der sozialen Dimension der EU beitragen könnten. Während eine kulturell-identitäre Solidarität sich für ihre Träger aus einer gemeinsamen Abstammung begründen würde, ergäbe sich eine demokratisch-legitimatorische Solidarität aus einem wechselseitig gültigen Rechtsvertrauen im Sinne liberal-demokratischer Werte. Als dritte Form benennen Loh und Skupien ${ }^{22}$ eine arbeitsteilig-wertschätzende Solidarität, welche sie »in den gegenseitigen Abhängigkeiten [...], die die moderne Arbeitsteilung mit sich bringt«, begründet sehen.

Um eine kooperations- und umverteilungsfeste Gemeinschaft unter den Bürgern der EU etablieren zu können, schreiben die Autoren den - ebenfalls im Durkheim'schen Sinne - intermediären Organisationen (das heißt Parteien, Verbänden, und hier vor allem den Gewerkschaften) das Potenzial zu, »als Transmissionsriemen für die Stärkung transnationaler arbeitsteiliger Solidarität zu wirken «. ${ }^{23}$ In diesem Sinne erkennen die Autoren aktuell auch nur die arbeitsteilig-wertschätzende Solidaritätsquelle als vielversprechenden Bezugspunkt zur Etablierung eines europäischen Gemeinwesens.

Jenseits des tarifpolitischen Tagesgeschäfts sehen die Autoren für die europäischen Gewerkschaften in diesem Prozess eine Protagonistenrolle: »Eine starke und funktionierende europäische Gewerkschaftsbewegung, institutionalisiert etwa in Form des Europäischen Gewerkschaftsbundes, kann als zentraler - oder zumindest als ergänzender - Akteur für eine weiter erstarkende europäische Öffentlichkeit und transnationale Parteien dienen. «24

18 In diesem Sinne ließe sich auch von einem Euro-Zweckoptimismus sprechen. Bemerkenswert erscheint weiterhin, dass der optimistische Diskurs wesentlich von Fachvertretern mitgetragen wird, die von den Gewerkschaften beschäftigt werden; vgl. Waddington, Hoffmann 2000; Kowalsky 2014; Schulten et al. 2005.

19 Loh, Skupien 2016, S. 578.

20 Hyman 2011.

21 Vgl. Durkheim 1966.

22 Loh, Skupien 2016, S. 585.

23 Ebd., S. 594.

24 Ebd. 
In der Praxis könnte sich diese neue Rolle innerhalb von zwei Dimensionen ausdrücken: Konkrete Erfolge in der tarifpolitischen Koordinierung oder im Lobbying gegenüber den europäischen Institutionen würden den Gewerkschaften aus dieser Sicht, erstens, unmittelbaren Einfluss auf die vertikale interessenpolitische Steuerung der Europäischen Union einräumen. Indem Gewerkschaften ihre alltägliche Vertretungsarbeit europäisierten, so die zweite Annahme, würde anhaltender Kontakt der Vertreter untereinander ein europäisches Bewusstsein im horizontalen Rahmen - etwa durch Bildungsarbeit oder auch gemeinsame Arbeitskämpfe auf Betriebsebene - stärken. Reale marktkorrigierende Effekte gingen so Hand in Hand mit vergemeinschaftungsstärkender Kooperation. Als erste Anzeichen für eine entsprechende Entwicklung erkennen die Autoren "spontane Solidareffekte « 25 in einem Item einer Survey-Auswertung, der zufolge ein europaweiter Mindestlohn die mehrheitliche Zustimmung europäischer Bürgerinnen und Bürger in Polen, Spanien und Deutschland findet - sogar dann, wenn eigene Einkommensverluste damit verbunden wären. ${ }^{26}$

Doch hierbei bleibt es nicht. Zum Zweck der graduellen Etablierung einer arbeitsteilig-wertschätzenden Solidarität innerhalb der EU schlagen Loh und Skupien ${ }^{27}$ konkrete »institutionelle und tarifpolitische Maßnahmen « in Form einer gemeinsamen Arbeitsmarktpolitik der EU-Staaten vor. Einen ersten wesentlichen Bezugsrahmen soll ihnen zufolge eine gemeinsame Arbeitslosenversicherung bieten. Im Zuge mitunter turbulenter Konjunkturdynamiken innerhalb des gemeinsamen Wirtschaftsraums soll eine europäische Arbeitslosenversicherung "quasi unter der Hand Transferleistungen innerhalb der Union ermöglichen, da in ökonomischen Krisenregionen tendenziell mehr Leute arbeitslos werden und dementsprechend dort nicht nur weniger in die Sozialsysteme einbezahlt, sondern auch vermehrt Arbeitslosengeld bezogen wird ".

Ihre Hoffnung, dass »eine Stärkung der europäischen Gewerkschaftsbewegung mittelfristig die vorherrschenden Konkurrenzdiskurse und Standortängste abmildern könnte " ${ }^{28}$ begründen die Autoren so mit der Aussicht auf die Ergänzung des nationalstaatlichen Bezugsrahmens durch »komplementäre Solidaritätsbeziehungen zwischen den Erwerbstätigen in ganz Europa « ${ }^{29}$ Nun ist gegen die These einer funktionierenden Tarifpolitik als Grundlage europäischer Solidarität - zumindest auf einer konzeptionellen Ebene - nichts einzuwenden. Denn wenn die Gewerkschaften nicht nur auf nationaler, sondern auch auf internationaler Ebene als effektive Lohnkartelle fungieren könnten, wären sie so in der Lage, den Unterbietungswettbewerb im gemeinsamen Wirtschaftsraum (und auch darüber hinaus) abzufedern. Doch inwiefern dies in der Praxis tatsächlich geschieht, steht natürlich auf einem anderen Blatt.

25 Ebd.

26 Gerhards, Lengfeld 2013, S. 210.

27 Loh, Skupien 2016, S. 599.

28 Ebd., S. 580.

29 Ebd., S. 598. 
Die Frage nach den Möglichkeiten grenzüberschreitender Solidarität bearbeiten die Autoren aus einer fast ausschließlich konzeptionellen Perspektive. Hierin liegt ein wesentliches Problem in der Argumentation. Denn die für die politische Praxis entscheidende Frage lautet ja nicht, ob Solidarität denkbar ist, sondern ob sie praktiziert werden kann. Ob europäische Gewerkschaften zu einem Modus solidarischer Kooperation gelangen und ob sich eine solche solidarische Kooperation auch tatsächlich in eine gemeinsame Identität als Voraussetzung einer umverteilungsfesten Wirtschaftsgemeinschaft überführen lässt, muss sich daher in der politischen Praxis herausstellen. Die im Folgenden zu präsentierenden Befunde sollen die realen Kapazitäten und Potenziale gewerkschaftlicher Zusammenarbeit in der EU auf empirischer Grundlage illustrieren.

\section{Theoretischer Bezugsrahmen und methodische Operationalisierung}

\subsection{Europäische Tarifpolitik als Gradmesser gewerkschaftlicher Positionsbildung}

Für die Frage, ob Gewerkschaften gemeinsame Positionen auf europäischer Ebene entwickeln können, rücken wir mit der Tarifpolitik den »wohl wichtigsten Maßstab für die Effektivität von Gewerkschaften « 30 in den Blick. Denn je stärker nationale Regulierungsmöglichkeiten im Integrationsprozess aufgeweicht oder abgeschafft werden, desto dringender stellt sich aus gewerkschaftlicher Sicht die Notwendigkeit, neue Instanzen der tarifpolitischen Regelsetzung zu etablieren. Unter Bedingungen des gemeinsamen Marktes und der Währungsunion müssten sich Maßnahmen einer solchen europäischen Tarifpolitik auf die Erhaltung oder den Ausbau nationaler oder transnationaler Arbeits- und Beschäftigungsstandards richten.

Kädtler ${ }^{31}$ zufolge umfasst das Feld der Tarifpolitik erstens »das Aushandlungssystem mit seinen Institutionen, Akteuren und Verfahren «, zweitens "das Feld der Themen und Gegenstände, die als tarifpolitisch reguliert bzw. in dieses Politikfeld einbezogen worden sind «, sowie drittens den »regulierende[n] Einfluss, der mit tarifpolitischen Mitteln auf dem Feld der Arbeits- und Entlohnungsbedingungen, ggf. aber auch darüber hinaus, entfaltet wurde oder wird «. Vor diesem Hintergrund erscheint Tarifpolitik als »das Ergebnis von Entscheidungsprozessen in Organisationen, in die äußere wie interne Rahmenbedingungen als Begrenzungen und als Voraussetzungen von Handeln eingehen ${ }^{3}{ }^{32}$

Tarifpolitik bezeichnet damit also nicht nur die Aushandlung tariflicher Standards, sondern auch die Regeln darüber, wie diese zu erfolgen hat, sowie deren Setzung und Neuverhandlung. Während zum einen substanzielle Fragen nach Lohn oder Arbeitsstandards geklärt werden, stehen gleichzeitig die Modi dieser Klärung zur Debatte. Die Abstimmung von Lohnabschlüssen zur Verhinderung einer Unterbietungskonkurrenz kann demzufolge hier genauso eine Rolle spielen wie die

30 Wiesenthal 1992, S. 7.

31 Kädtler 2013, S. 427.

32 Ebd., S. 428. 
gemeinsame Formulierung und Durchsetzung bestimmter Mindeststandards im europäischen Rahmen.

Traditionell findet die tarifpolitische Positionsbildung europäischer Gewerkschaften im symbolischen Bezugsrahmen des »Europäischen Sozialmodells « statt. Der Begriff eines "Sozialen Europa " hat seinen Ursprung in der politischen Diskussion der frühen 1970er Jahre ${ }^{33}$ und gewinnt seine ersten Konturen maßgeblich unter der Federführung des ehemaligen Kommissionsvorsitzenden Jacques Delors. Vor dem Hintergrund der EU-Osterweiterung verweist die Konzeption eines "Sozialen Europa « auf die Hoffnung der politischen Linken, »jenes reuropäische Sozialmodell erhalten zu können, das sich durch eine sozial regulierte Variante des Kapitalismus auszeichnet, die auf eine Verbindung von ökonomischer Effizienz und (relativer) sozialer Gleichheit zielt «. ${ }^{34}$

Als "gesellschaftspolitische Zukunftsformel « beschreibt der Begriff eines "Europäischen Sozialmodells « Aust et al. ${ }^{35}$ zufolge "ein politisch-ideologisches Konstrukt [...], das europäische Gemeinsamkeiten definiert und propagiert, die erst noch zu realisieren wären «. Mit welchen Schwierigkeiten dabei zu rechnen sein wird, zeigt die Studie, deren methodische Konzeption und forschungspraktische Operationalisierung hier kurz skizziert werden.

\subsection{Methodische Operationalisierung}

Die im Folgenden verwendeten Daten stammen aus einem Projekt zur Untersuchung der gewerkschaftlichen Positionsbildung im Bereich der europäischen Tarifpolitik. ${ }^{36} \mathrm{Um}$ möglichst allgemeine Befunde zu gewährleisten, wurden hierzu die drei wichtigsten Felder ausgewählt, namentlich die Auseinandersetzung um die Dienstleistungsfreiheit, die Diskussion zum europäischen Mindestlohn sowie die Initiativen zur Lohnkoordinierung im gemeinsamen Währungsraum. Alle drei Politikfelder gewinnen eine besondere Bedeutung durch die Vertiefung und Erweiterung der europäischen Integration.

Kritische Beiträge wie die von Höpner und Lutter ${ }^{37}$ oder auch Scharpf ${ }^{38}$ gelangen über die Potenziale der Positionsbildung politischer Akteure in der EU aus einer strukturvergleichenden Perspektive zu pessimistischen Schlussfolgerungen. Diese Argumentation betont die Schwierigkeiten, vor die sich europäische Gewerkschaften bei der Etablierung gemeinsamer Positionen in der Tarifpolitik gestellt sehen. Doch hier bleiben, so eine Kritik von Pernicka, ${ }^{39}$ die "transnationalen Austauschbeziehungen zwischen arbeitspolitischen Akteuren [...] unterbelichtet «. Wie die (für die Etablierung gemeinsamer Positionen vermeintlich inkompatiblen) Rahmenbe-

33 Pierson, Leibfried 1998.

34 Schulten 2005, S. 15.

35 Aust et al. 2002, S. 273.

36 Seeliger 2017.

37 Höpner, Lutter 2014.

38 Scharpf 2008.

39 Pernicka 2015, S. 7. 
dingungen sich auf das politische Handeln der politischen Akteure tatsächlich auswirken, kann demnach erst eine handlungstheoretische und akteurzentrierte Sichtweise zum Vorschein bringen.

Im Sinne eines solchen Zugangs verfolgte die Datenerhebung das Ziel einer Rekonstruktion davon, »wie Akteure die Dynamik der Europäischen Integration interpretieren, sich zu eigen machen oder ignorieren « ${ }^{40}$ Hieraus ergab sich die Notwendigkeit eines qualitativen Forschungsdesigns. Die Datengrundlage bilden 87 halbstandardisierte, im Zeitraum zwischen Oktober 2013 und Juni 2015 durchgeführte Experteninterviews mit Gewerkschaftern ${ }^{41}$ aus sieben Ländern (Deutschland, Schweden, Norwegen, Polen, Ungarn, Italien, Spanien) sowie der EU-Ebene.

\section{Europäische Tarifpolitik im Integrationsprozess - Empirische Befunde}

Europessimistischen Annahmen gegenüber erscheinen die Befunde aus den drei ausgewählten Politikfeldern zumindest als überraschend. Denn es ist der politökonomischen Heterogenität sowie der Schwäche der europäischen Verbände zum Trotz in der grenzüberschreitenden Zusammenarbeit europäischer Gewerkschaften zur Etablierung gemeinsamer Positionen gekommen. Ob die Optimisten mit ihren konzeptionellen Argumenten also doch Recht behalten? Eine Antwort hierauf soll die folgende Analyse der genannten drei wichtigsten Felder der europäischen Tarifpolitik im Zeitraum der letzten anderthalb Jahrzehnte geben.

Im Bereich der Dienstleistungsfreiheit sowie hinsichtlich der Diskussion um den europäischen Mindestlohn ergibt sich diese Heterogenität vor allem aus den Unterschieden mit Blick auf die Lohnhöhe und die Arbeits- und Beschäftigungsstandards. Diskrepanzen zwischen den weniger regulierten Arbeitsmärkten der mittel- und osteuropäischen Länder auf der einen und den hochregulierten nord- und westeuropäischen Ländern resultieren hier aus unterschiedlichen Entwicklungspfaden über die letzten Jahrhunderte. ${ }^{42}$ Die besondere tarifpolitische Bedeutung der EU-Osterweiterungen der Jahre 2004 und 2007 zeigt sich auch im Vergleich mit der SüdErweiterung in den 1980er Jahren. Während das Pro-Kopf-Einkommen von Griechenland und Portugal immerhin bei zwei Dritteln des damaligen EU-9Durchschnitts lag, betrug es bei der ersten Erweiterungsrunde 2004 nur 45 Prozent und 2007 bei Bulgarien und Rumänien nur ein Drittel. ${ }^{43}$

Die Untersuchung der ersten beiden Politikfelder erfolgt hier unter Bezug auf drei Länder, die unterschiedliche (wenn auch nicht alle) Regionen der EU repräsentieren. Als Vertreter des "Nordic Model « ${ }^{44}$ repräsentieren die schwedischen Gewerkschaf-

40 Woll, Jacqout 2010, S. 118.

41 Zum Zweck der Anonymisierung (und nicht, weil alle Interviewten Männer waren) wird - ungeachtet des realen Geschlechts der jeweiligen Personen - im Folgenden immer die männliche Form verwendet.

42 Meardi 2012, S. 16.

43 Krings 2015, S. 90.

44 Hilson 2008. 
ten ein Spektrum der auf europäischer Ebene etablierten und einflussreichen Organisationen. Die Auswahl polnischer und ungarischer Gewerkschaftsorganisationen als Repräsentanten der mittel- und osteuropäischen Länder ergibt sich aus der Tatsache, dass deren Einfluss auf der nationalen und internationalen Ebene heute allgemein als sehr schwach angesehen wird. Tabelle 1 zeigt eine Reihe wesentlicher tarifpolitischer Indikatoren für die drei Länder.

Tabelle 1: Rabmendaten zu den Tarifsystemen der untersuchten Länder ${ }^{45}$

\begin{tabular}{|l|c|c|c|}
\hline & $\begin{array}{c}\text { Flächentarifliche } \\
\text { Deckungsrate }\end{array}$ & $\begin{array}{c}\text { Gewerkschaftlicher } \\
\text { Organisationsgrad }\end{array}$ & $\begin{array}{c}\text { Gesetzlicher } \\
\text { Mindestlohn }\end{array}$ \\
\hline Schweden & $88 \%$ & $70 \%$ & - \\
\hline Polen & $25 \%$ & $12 \%$ & 2,21 Euro \\
\hline Ungarn & $33 \%$ & $12 \%$ & 1,95 Euro \\
\hline
\end{tabular}

Mit Blick auf ihr europäisches Engagement lassen sich unter den nord- und osteuropäischen Gewerkschaften ebenfalls unterschiedliche "national approaches to internationalism « 46 verzeichnen: Evidenz für eine entsprechend integrationsfreundliche Haltung unter den osteuropäischen Vertretern findet Pleines ${ }^{47}$ auf Basis einer Erhebung unter Gewerkschaftern aus Polen, Tschechien und der Slowakei. Etwa zwei Drittel der Befragten geben an, dass sie die allgemeine politische Bedeutung der EU gegenüber den Nationalstaaten momentan als zu gering ansehen. Gleichzeitig überträgt sich auf Seiten der Nordeuropäer ein Vertrauen in die Überlegenheit des nordischen Regimes der Industriebetriebsziehungen ${ }^{48}$ in einen institutionellen Nationalismus « ${ }^{49}$, der den Schutz der eigenen Systeme vor einer Verlagerung sozialund tarifpolitischer Kompetenz auf die europäische Ebene zum zentralen strategischen Bezugspunkt werden lässt.

\subsection{Die Auseinandersetzung um die Dienstleistungsfreiheit}

In der Auseinandersetzung um die Dienstleistungsfreiheit ergibt sich die Herausforderung für die Gewerkschaften vor allem aus den Konsequenzen der Erweiterungsrunden der Jahre 2004 und 2007. Ein Ost-West-Gefälle im Hinblick auf Wohlstand, Lohnkosten und Arbeitsrecht schaffte eine Anreizstruktur für die neuen Mitgliedsländer, westliche Standards im gemeinsamen Markt zu unterbieten. Die jeweiligen nationalen Hintergründe und Traditionen der hier untersuchten Gewerkschaften aus Schweden, Polen und Ungarn spiegeln die dominante Konfliktlinie zwischen ost-

45 Schulten 2013.

46 Gumbrell-McCormick, Hyman 2013, S. 162.

47 Pleines 2008, S. 18.

48 Götz, Haggrén 2009, S. 15.

49 Streeck 1998. 
und westeuropäischen Ländern. Schweden steht hierbei repräsentativ für eine Reihe vor allem westeuropäischer Arrangements der Arbeitsbeziehungen mit hohen Lohnund Beschäftigungsstandards (Deutschland, Italien, Frankreich, die BeNeLuxLänder, Großbritannien und Skandinavien). Im Kontrast zum hohen Grad an Regulierung, dem die Arbeitsbeziehungen in diesen Ländern unterliegen, sind entsprechende Standards in den neuen Mitgliedsländern schwächer ausgeprägt. Aus dieser Diskrepanz ergab sich eine Konstellation, in der für die mittel- und osteuropäischen Lohnabhängigen ein struktureller Anreiz besteht, durch die Unterschreitung dieser Standards einen Wettbewerbsvorteil zu erwirken.

Die Dienstleistungsfreiheit ist eines der Prinzipien des gemeinsamen europäischen Marktes. Die entscheidenden Weichenstellungen in diesem Politikfeld ereigneten sich im Zeitraum von 2004 bis 2006 im Zuge der EU-Osterweiterung. Mit der Dienstleistungsrichtlinie schlug das Generaldirektorat Binnenmarkt und Dienstleistungen unter der damaligen Leitung von Frits Bolkestein eine Richtlinie vor, welche mit dem Herkunftslandprinzip eine Regelung enthielt, die das Territorialitätsprinzip tariflicher Standards infrage stellte. Durch die Beschäftigung ausländischer Dienstleister sollte es so im gemeinsamen Markt möglich werden, schwächere Lohn- und Arbeitsstandards zu importieren. Das wachsende Lohn- und Wohlstandsgefälle und die Unterschiede im Arbeitsrecht, welche die EU nach ihrer Erweiterung nach Osten hin auszeichneten, beinhalteten für die europäischen Gewerkschaften ein großes Konfliktpotenzial. Wie sollte man den osteuropäischen Gewerkschaften erklären, dass sie diese Diskrepanz nicht etwa als strukturellen Vorteil in einem europäischen Unterbietungswettbewerb nutzen sollten?

Nun gilt die Auseinandersetzung um die sogenannte Bolkestein-Richtlinie im Spiegel der Forschung gemeinhin als Fall gelungener Zusammenarbeit, in dem Gewerkschaften aus Ost und West trotz unterschiedlicher Hintergründe eine gemeinsame Position haben entwickeln und in eine gemeinsame Kampagne überführen können, an deren Ende das Herkunftslandprinzip aus der Richtlinie entfernt werden konnte. ${ }^{50}$ Mit Blick auf das Zustandekommen dieser Position werfen die hier vorzustellenden Befunde allerdings eine Reihe von Fragen an die praktische Ausgestaltung des Konzepts "Soziales Europa « auf.

Für den Ausgangspunkt der Diskussion erkennt ein ehemaliger Vertreter des Europäischen Gewerkschaftsbunds (EGB) eine allgemeine »fear from the hordes of Eastern Europe «, welche auch ein Repräsentant der Europäischen Föderation der Bau- und Holzarbeiter bestätigt:

"Ich meine, die ganzen osteuropäischen Kollegen mit ihren Gewerkschaftsstrukturen. Und ihren schlecht ausgebauten Verträgen und Kontrollsystemen und relativ tiefen Sozialstandards, in den EU-Markt aufgenommen werden, dass da natürlich die ganze Diskussion des Lohndumpings ganz neu losgeht, ist ja nicht ganz falsch.«

Angesichts der tarifpolitischen Bedrohung, die nord- und westeuropäische Vertreter in der Dienstleistungsrichtlinie erkennen, erscheint es wenig überraschend, dass sie ihre Interessen gegenüber den Repräsentanten der osteuropäischen Gewerkschaften

50 Die Kampagne fand ihren Höhepunkt in einer Demonstration mit 75.000 Teilnehmern am 19. März 2005, unter ihnen zahlreiche Vertreter der osteuropäischen Verbände. 
mit einiger Vehemenz vertreten. Die Diskussion fand im transnationalen Rahmen statt, das heißt zwischen den Brüsseler Zusammenkünften der nationalen Delegierten und in den jeweiligen Hauptquartieren der nationalen Mitgliedsorganisationen des EGB. Reibung und Konflikt bei der Etablierung der Position beschreibt der Vertreter der ungarischen Föderation MSZOSZ (Magyar Szakszervezetek Országos Szövetsége). Ihm zufolge sei die Anerkennung westlicher Standards unter ungarischen Gewerkschaftern keineswegs von vornherein als Selbstverständlichkeit angesehen worden:

"Wir hatten auch Konflikte unter uns hier in diesem Saal, wo ich und einige angegriffen wurden von unseren Gewerkschaften, dass ... Es ist besser, wenn man weniger als der Einheimische verdient, aber immerhin es gibt Arbeitsplatz, da kommt viel mehr als zu Hause."

Zum Verständnis der Konstellation, innerhalb derer diese Debatte geführt wurde, erscheint es unverzichtbar, sich über die Diskrepanzen und Etabliertenvorrechte im Klaren zu sein, wie sie die grenzüberschreitende Abstimmung zwischen Gewerkschaftern aus den alten und den neuen Mitgliedsländern im Verlauf der letzten zwei Jahrzehnte wesentlich geprägt haben. Diese Konstellation tritt etwa in der folgenden Passage desselben Vertreters zutage: "Wir sind dazugekommen. Das ist so, das ist normal. Da ist schon eine Familie und Du kommst von außen. Das ist ganz verständlich."

Die Metapher der Familie als Sphäre nicht instrumenteller, sondern affektiv motivierter Kooperation erweitert den Interpretationsspielraum in einen Bereich jenseits materieller Komponenten hinein - es geht um Anerkennung. Und diese sieht der ungarische Gewerkschafter in der Auseinandersetzung als nicht gewährleistet. Eine komplementäre Einschätzung äußert ein Vertreter der deutschen IG BAU als Antwort auf die Frage, ob die Vertreter aus den östlichen Beitrittsländern eigentlich souveräne Europäer seien: "Ne, sehe ich nicht so. Also, viele haben Europa als solches, EU, mit all seiner Widersprüchlichkeit und Brüchigkeit eigentlich noch gar nicht richtig begriffen. "Über die Fähigkeit, sich souverän am Betrieb der gewerkschaftlichen Europapolitik zu beteiligen, verfügten diese daher nur in begrenztem Maße.

Gleichzeitig lassen sich auch die hier dargestellten Ausführungen der osteuropäischen Vertreter mit Blick auf das Feld der Dienstleistungsfreiheit zu einem Schluss verdichten, den ein polnischer Vertreter des EGB besonders griffig formuliert. Das "Soziale Europa « ist ihm zufolge »ziemlich offensichtlich eine westliche Idee «. Entwickelt von westeuropäischen Vertretern mit westeuropäischen Maßstäben, sahen sich die Osteuropäer dieser Darstellung zufolge einem abgeschlossenen Konzept gegenübergestellt, das sie nun akzeptieren sollten. Eine gemeinsame politische Linie ergibt sich hier also über die Einwilligung der Vertreter aus den neuen Mitgliedsländern in eine Position, an deren ursprünglicher Entstehung sie gar nicht beteiligt waren.

\subsection{Europäischer Mindestlobn}

Die Diskussion um einen europäischen Mindestlohn mag, oberflächlich betrachtet, wenig kontrovers erscheinen - welche Gewerkschaft sollte schon gegen eine Lohn- 
untergrenze votieren? Ein genauerer Blick auf die Gegebenheiten der nationalen Systeme industrieller Beziehungen in den skandinavischen Ländern zeigt jedoch, dass vergleichsweise hohe Löhne und gute Arbeitsbedingungen dort unter Bedingungen absoluter Tarifautonomie erreicht werden. Aus Sicht der skandinavischen Gewerkschaften musste eine europäische Mindestlohnregelung die Verhandlungsautonomie von Arbeitgebern und Gewerkschaften in der Tarifpolitik gefährden und möglicherweise perspektivisch zu einem Bedeutungsverlust letzterer beitragen.

Wie Schulten et al..$^{51}$ in einem frühen programmatischen Beitrag festhalten, ist das Ziel hierbei also keinesfalls eine absolute Untergrenze: "Bei dem Konzept einer europäischen Mindestlohnpolitik handelt es sich demnach im Kern um die europaweite Festlegung bestimmter gemeinsamer Ziele und Kriterien, auf deren Grundlage dann die nationalen Mindestlohnpolitiken miteinander koordiniert werden können. «

Konkret fordert man im Sinne dieses Konzepts die Einrichtung einer nationalen Lohnuntergrenze in Höhe von erst 50 Prozent und später 60 Prozent der nationalen Medianlöhne, nicht auf Ebene der EU, sondern durch die nationalen Regierungen. Nachdem die Forderung an verschiedenen Stellen (unter anderem in den Manifesten zu den EGB-Kongressen in Sevilla und Athen der Jahre 2007 und 2011) geäußert und durch die Debatte um die European Economic Governance angeheizt worden war, verabschiedete man den Vorschlag zur Festlegung der geforderten Lohnuntergrenze in Höhe von 50 Prozent und später 60 Prozent der nationalen Medianlöhne schließlich auf der EGB-Winterschool Anfang des Jahres 2012 in Kopenhagen:

"The ETUC recommends that where it exists the effective national minimum wage should be at least equal to $50 \%$ of the average wage or $60 \%$ of the median wage. ETUC actively supports its affiliates in their actions to gradually reach this goal, in accordance with their national circumstances. Countries which have already achieved this goal should aim for a more ambitious target. «52

Diese Position sollte laut dem dortigen Beschluss in einer Kampagne nach außen getragen und offensiv vertreten werden. Während man im Rahmen des EGB zwar eine nominelle Einigung über eine Regelung zum europäischen Mindestlohn erzielen konnte, wurde eine entsprechende Kampagne bis heute (März 2017) noch nicht umgesetzt.

Exemplarisch stehen die schwedischen Vertreter im Untersuchungsdesign für eine breitere Opposition gegen einen allgemeingültigen europäischen Mindestlohn, die neben den skandinavischen außerdem die österreichischen und italienischen Gewerkschaften einschließt. Auf der anderen Seite engagieren sich Gewerkschafter aus Polen und Ungarn seit Beginn der Diskussion für eine europäische Lohnuntergrenze. Aus dieser Sicht soll die europäische Ebene als Hebel für die nationale Lohnpolitik genutzt werden. Die schwedischen Vertreter nennen im Wesentlichen drei Gründe für ihre Ablehnung: Zum einen würde eine solche Regelung die Verhandlungsposition der Skandinavier nicht stärken, da die Löhne ohnehin recht hoch ausfallen. Zweitens möchte man den Staat aus der Lohnfindung heraushalten, um

51 Schulten et al. 2005.

52 EGB 2012, S. 8. 
die eigene lohnpolitische Kompetenz (und damit das tarifpolitische Kerngeschäft) nicht aus der Hand zu geben. Und drittens ist man der europäischen Arena gegenüber allgemein skeptisch eingestellt und befürchtet, auf diese Weise perspektivisch die eigene Autonomie aufzugeben.

Eine angespannte Atmosphäre bei der Diskussion des Vorschlags beschreibt ein Vertreter der polnischen OPZZ (All-Poland Alliance of Trade Unions):

"We have had a kind of informal meeting in London, and the president of all the Nordics, of FTF white collar workers, she was really defending this. And our president and her, there was a clash. The language was hostile. Both stepped forward on both sides. "

Eine eher undiplomatische Haltung spricht auch aus folgender Äußerung eines Vertreters der schwedischen Verbands LO (The Swedish Trade Union Confederation):

"The moment someone in a capacity as an ETUC official has a say that the ETUC supports the European minimum wage, we will do our best to sack that person. Because that person will work directly against our interest. We do not want intervention in our wage issues."

Erneut ist es also vor allem die Machtasymmetrie im Rahmen der europäischen Verbände zu Ungunsten der osteuropäischen Kollegen, die die offene Proklamierung der Notwendigkeit eines europäischen Mindestlohns verhindert. Eine gemeinsame Position konnte zwar formuliert, allerdings nicht in die Praxis übertragen werden.

Ähnlich wie schon im Fall der Diskussion um die Dienstleistungsfreiheit überträgt sich ein Machtungleichgewicht auf europäischer Ebene in die Agenda der europäischen Tarifpolitik. Diesen Umstand bedauert ein Repräsentant der ungarischen MSZOSZ: »Aber sehr oft, wir haben das Gefühl, dass auch der EGB beschäftigt sich hauptsächlich mit Fragen der Westeuropäer. « Etabliertenvorrechte und Ressourcendiskrepanz bedingten ihm zufolge weiterhin eine Konstellation, innerhalb derer die Osteuropäer systematisch unterlegen seien:

"Das ist überhaupt nicht balanciert, wir [die Osteuropäer; M.S.] sind Neulinge. Obwohl, wenn man 20 Jahre dabei ist, ist man schon nicht mehr Neuling. Aber auf unserer Seite sehen wir, dass wir schwach sind. Wir sind schwach allgemein und haben auch kaum echte Ressourcen, alle Politikbereiche wirklich zu bearbeiten und offensiv in die Diskussionen reinzugehen.

Das "Soziale Europa " erscheint also in der Praxis in erster Linie als tarifpolitisches Projekt zur Sicherung westeuropäischer Standards. Von einer solidarischen Zusammenarbeit auf Basis der Unterstützung der Anliegen weniger einflussreicher Gewerkschaften durch die stärkeren kann hier also keine Rede sein.

\subsection{Initiativen zur europäischen Lobnkoordinierung}

Die politische Auseinandersetzung um den Lohn erfährt unter Bedingungen der Währungsunion insgesamt einen signifikanten Bedeutungszuwachs. ${ }^{53}$ Bei anpassbaren Wechselkursen konnten die Länder mit Wechselkurskorrekturen auf inländische Lohn- und Preissteigerungen reagieren. Unter Bedingungen des gemeinsamen Währungsregimes ist das nun nicht mehr möglich. Für die ließe sich daher ein besonderer Anreiz vermuten, über eine absichtlich moderat gestaltete Lohnentwicklung den Erhalt von Arbeitsplätzen im Land sicherzustellen. Tatsächlich unterhalten

53 Streeck 2013. 
die Gewerkschaften des Metallsektors aber seit Mitte der 1990er Jahre Initiativen zur Koordinierung der Lohnabschlüsse im europäischen Binnenmarkt mit dem Ziel, einen solchen Unterbietungswettbewerb zu verhindern. ${ }^{54}$ Die Beibehaltung dieser Initiativen erscheint angesichts der neuen Wettbewerbskonstellation im Euro-Raum zumindest als erklärungsbedürftig.

Die besondere Rolle der IG Metall innerhalb dieser Koordinierungsinitiativen ergibt sich aus der Bedeutung des deutschen Metallsektors als wichtigstem Exportsegment der europäischen Wirtschaft. Von den ca. 40 Prozent des gesamten deutschen Außenhandels mit den Ländern der Euro-Zone geht der Großteil von der Metall- und Elektroindustrie aus. So ist erstens anzunehmen, dass in diesem Sektor die internationale Konkurrenz am stärksten ist. ${ }^{55}$ Zweitens sind die Koordinierungsinitiativen hier am weitesten fortgeschritten. Und drittens ergibt sich in der Exportwirtschaft aufgrund des größten Außenhandelsvolumens im sektoralen Vergleich der potenziell stärkste Einfluss auf die Makroökonomie innerhalb der EuroZone.

Gleichzeitig besteht, so ließe sich argumentieren, zumindest in den Exportsektoren ein Anreiz für die Gewerkschaften, den Verhandlungsspielraum nicht völlig auszuschöpfen, um die nationale Wettbewerbsfähigkeit der Mitglieder gegenüber ausländischen Konkurrenten zu verbessern. Um diesem Problem zu begegnen, haben die Gewerkschaften der Metallsektoren vereinbart, eine Lohnentwicklung im Einklang mit Produktivitäts- und Inflationsentwicklung anzustreben. Das Prinzip dieser Regelung ist in den Publikationen dieser Gewerkschaften und auch des europäischen Dachverbands niedergelegt. Diese Lohnformel (mindestens Produktivitätszuwachs plus Inflationsrate) findet sich in der "Politischen Resolution 2011-2015 « des neu gegründeten Verbands IndustriALL Europe ${ }^{56}$ und wird bekräftigt von der im Juni 2014 in Wien abgehaltenen tarif- und sozialpolitischen Konferenz der Organisation:

"Lohnerhöhungen, die über den Produktivitätszuwachs und Inflationsausgleich hinausgehen, sind wirtschaftlich sinnvoll und sozial verantwortlich, weil eine Umverteilung von Kapital- zu Lohneinkommen der beste Weg zur Konjunkturbelebung ist. « ${ }^{57}$

An anderer Stelle ${ }^{58}$ wurde vom Verfasser dieses Beitrags ausgerechnet, inwiefern die Tarifabschlüsse der IG Metall dieser Koordinierungsregel im Verlauf der letzten 15 Jahre entsprochen haben. Von der Einführung eines festen Wechselkurses im Jahre 1998 bis zum Jahr 2013 liegt die kumulierte Negativabweichung im Tarifvertrag der Metall- und Elektroindustrie bei 2,9 Prozent beziehungsweise 3,3 Prozent, je nachdem, ob man die nationale Inflationsrate oder die Zielinflationsrate der EZB zum Maßstab nimmt. Praktisch wurde das Ziel der Lohnformel also nicht erreicht.

54 Glassner 2009.

55 Hancké 2013.

56 IndustriALL 2012, S. 13; IndustriALL 2014.

57 IndustriaLL 2014.

58 Seeliger 2017, Abschnitt 5.4. 
Im Rahmen der Interviews erklärt einer der befragten IG-Metall-Vertreter die praktische Relevanz der Koordinierungsregel mit den folgenden Worten:

"Also, der Begründungszusammenhang, den man eigentlich verinnerlicht hat, [ist] auch nach außen hin so darzustellen. Und dann hat es, wenn wir in der Bewegung sind, nicht mehr so eine hohe Bedeutung. Also, Du kannst Dich beim Warnstreik da hinstellen und sagen, wir machen es deshalb, weil unsere europäischen Freunde das benötigen."

Und ganz ähnlich beschreibt dies sein Kollege aus einem anderen Tarifgebiet:

"Europa ist da nicht so präsent, wie sich das vielleicht in den Broschüren, oder wie man sich das in der Wirklichkeit so vorstellt. Wie gesagt, da sitzt keiner im Hinterzimmer und hat den europäischen Rechner auf, tippt das ein und sagt, geht nicht, oder geht. Sondern das ist eher so ein Punkt, wo man sagt, wir müssen uns auch dazu rechtfertigen, wir müssen das einfließen lassen, aber in der praktischen Arbeit habe ich jetzt keinen Ordner in der Tarifrunde 2015, wo ich jetzt Europa stehen habe.

Dass es Lohnkoordinierung gibt, so lässt sich hier schließen, ist also - wenigstens der Außendarstellung nach - eine Angelegenheit von großer tarifpolitischer Bedeutung. Andererseits wird sie nicht konsequent in ihrem nominellen Sinne verfolgt. Die beteiligten Organisationen betreiben sie trotzdem, um äußeren Erwartungen gerecht zu werden und ihre Handlungsfähigkeit mit Blick auf eine Koordinierung in der Zukunft zu erhalten.

\subsection{Zusammenfassung}

Wie die Studie zeigt, sind die Etablierung und Umsetzung gemeinsamer Positionen für Gewerkschaften im Bereich der europäischen Tarifpolitik schwierig. Tabelle 2 illustriert die Konstellation in den drei benannten Feldern.

Tabelle 2: Ergebnisse gewerkschaftlicher Positionsbildung in der europäischen Tarifpolitik

\begin{tabular}{|l|l|l|l|l|}
\hline & $\begin{array}{l}\text { Nominelle Position } \\
\text { der Gewerkschaf- } \\
\text { ten }\end{array}$ & Umsetzung & Ergebnis & Ursachen \\
\hline $\begin{array}{l}\text { Dienstleistungs- } \\
\text { freiheit }\end{array}$ & $\begin{array}{l}\text { Dienstleistungs- } \\
\text { richtlinie }\end{array}$ & Ja & $\begin{array}{l}\text { Interesse der westeuro- } \\
\text { päischen Verbände; } \\
\text { übertragen auf osteu- } \\
\text { ropäische }\end{array}$ & $\begin{array}{l}\text { Überzeugungskraft } \\
\text { der etablierten westeu- } \\
\text { ropäischen Vertreter } \\
\text { gegenüber den unterle- } \\
\text { genen osteuropäischen } \\
\text { Repräsentanten }\end{array}$ \\
\hline $\begin{array}{l}\text { Europäischer } \\
\text { Mindestlohn }\end{array}$ & Ja & Nein & $\begin{array}{l}\text { Gegner blockieren } \\
\text { Umsetzung der Posi- } \\
\text { tion, daher keine ent- } \\
\text { sprechende Kampagne }\end{array}$ & $\begin{array}{l}\text { Übermacht der Min- } \\
\text { destlohn-Gegner ver- } \\
\text { hindert Umsetzung } \\
\text { eines zumindest rheto- } \\
\text { rischen Kompromisses }\end{array}$ \\
\hline $\begin{array}{l}\text { Lohnkoordinie- } \\
\text { rung }\end{array}$ & Ja & Nein & $\begin{array}{l}\text { Einfluss bleibt bei } \\
\text { nationalen Verbänden } \\
\text { und allgemein } \\
\text { schwach }\end{array}$ & $\begin{array}{l}\text { Rhetorische Entspre- } \\
\text { chung bei praktischer } \\
\text { Abweichung }\end{array}$ \\
\hline
\end{tabular}


Die Diskussion um die Dienstleistungsrichtlinie zeigt hierbei das einzige positive Ergebnis: Obwohl das Wohlstandsgefälle sowie Unterschiede hinsichtlich der Arbeits- und Sozialstandards zwischen alten und neuen Mitgliedsländern die Akzeptanz einer geteilten politischen Linie unwahrscheinlich erscheinen ließen, gelang die Entwicklung einer konsensualen politischen Haltung jenseits des nationalen Bezugsrahmens. Bei genauerem Hinsehen zeigt sich allerdings, dass die Beteiligten (nicht nur die osteuropäischen) in der prozeduralen Erreichung dieses Ziels wesentliche Defizite erkennen - die Diskussion ist nicht auf Augenhöhe geführt worden. Die gemeinsame Position kommt, dies zeigen die Äußerungen der Interviewpartner, also zum Preis einer unausgeglichenen Etablierung. Dieselbe Machtasymmetrie kommt auch in der Diskussion um den europäischen Mindestlohn zum Tragen. Während die Vertreter der nationalen und internationalen Verbände hier zwar eine nominelle Einigung auf eine gemeinsame Kampagne erreichen konnten, scheiterte die Umsetzung an einer Initiative durch die skandinavischen Repräsentanten. Die Spezifika nationaler Interessenlagen sind mit einer gemeinsamen Position inkompatibel. Eine ähnliche Lage findet sich auch im Feld der Lohnkoordinierung. Nachdem man sich vor allem vor dem Hintergrund der Währungsunion auf die Lohnformel zur Verhinderung von Unterbietungswettbewerb geeinigt hat und eine Reihe ritueller Verfahrensweisen etabliert hat, um ihr zumindest zeremoniell zu entsprechen, zeigt sich kein realer Koordinierungseffekt. Eine Erklärung hierfür liefern die Aussagen der Interviewpartner, die die praktische Bedeutung der Regel deutlich relativieren.

\section{Fazit}

Ausgehend von einer Diskussion der Vorschläge von Loh und Skupien, ${ }^{59}$ die eine Stärkung der europäischen Gewerkschaftsbewegung sowie eine Europäisierung der nationalen Arbeitslosenversicherungen als Fundamente grenzüberschreitender Solidarität innerhalb der EU proklamierten, wurde hier gezeigt, wie schwierig die gewerkschaftliche Positionsbildung in den drei wichtigsten Feldern der europäischen Tarifpolitik ist. Die Tatsache, dass eine gemeinsame Position hier ausschließlich im Bereich der Dienstleistungsfreiheit (und dies lediglich unter Bedingungen einer Machtasymmetrie zwischen den beteiligten Lagern) etabliert und praktisch umgesetzt werden konnte, lässt unter den gegebenen Bedingungen ernsthafte Zweifel an der Realisierbarkeit dieser konzeptionellen Vorschläge aufkommen. Wenn die Autoren etwa proklamieren, dass eine "starke und funktionierende europäische Gewerkschaftsbewegung [...] als zentraler - oder zumindest als ergänzender Akteur für eine weiter erstarkende europäische Öffentlichkeit und transnationale Parteien dienen « 60 könne, lässt sich das nur als eine Tautologie begreifen, die (bisher) nur so lang funktioniert, wie wir sie nur als konzeptionelle Erwägung und nicht als praktischen Maßstab betrachten.

59 Loh, Skupien 2016.

60 Ebd., S. 589. 
Die hier vorgestellten Befunde deuten zudem darauf hin, dass die handlungsleitende Maxime im Bereich der Tarifpolitik keineswegs die von den Autoren betonte gemeinsame europäische Solidarität darstellt. Die Orientierungen der beteiligten Akteure in den Bereichen der Tarifpolitik erscheinen trotz arbeitsteilig begründeter Interdependenzen im gemeinsamen Markt als in erster Linie machtpolitisch und selbstreferenziell und eben nicht solidarisch motiviert ${ }^{61}$ Die Mobilisierung des Deutungsrahmens eines "Sozialen Europa " dient in den ersten beiden untersuchten Bereichen der Wahrung von Gewerkschaftsinteressen aus den alten Mitgliedsländern. ${ }^{62}$ Die These einer solidarischen Zusammenarbeit im Sinne einer Stärkung schwacher Interessen durch stärkere steht angesichts der Ergebnisse infrage.

Dass die westeuropäischen Vertreter ihre osteuropäischen Kollegen letztlich mit der Begründung überzeugen konnten, dass die Angleichung der Sozialstandards die Einstellung des Unterbietungswettbewerbs erfordern würde, birgt hierbei eine interessante Parallele zu den Bestrebungen der Lohnkoordinierung, die von ihren Protagonisten ebenfalls als zukunftsträchtiges Projekt dargestellt werden. Und hierfür gibt es auch einen guten Grund: Ein, wenn nicht das zentrale Problem für EU-Tarifpolitik ergibt sich aus der Tatsache, dass Arbeitnehmerinteressen häufig ohne groBen zeitlichen Horizont vertreten werden müssen: »Die Prekarität der Arbeitsverhältnisse verleitet die Lohnabhängigen dazu, sich auf das Nächstliegende zu konzentrieren, die Langfristperspektive zu verdrängen, derer es bedürfte, um eine Herausforderung für den Kapitalismus zu bieten. «63

Die europäische Integration ist aber ein langfristig angelegtes Projekt, welches sich - auch im Sinne seiner sozialdemokratisch-sozialistischen Fürsprecher - jenseits solcher unmittelbaren Bedürfnisse vollzieht. Die Konstruktion eines europäischen Wohlfahrtsstaates mit einer territorial konsistenten Arbeitspolitik sowie (annähernd) gleichen Lebensstandards ist ein Projekt, dessen Verwirklichung auch nach jahrzehntelanger Entwicklung nicht garantiert werden könnte. Die Interessen der Lohnabhängigen sind jedoch an die Befriedigung ihrer Bedürfnisse in der Gegenwart gebunden. Um diese Kurzfristperspektive als Leitorientierung gewerkschaftlicher Politikorientierung überwinden zu können, bedarf es überzeugender Argumente. Inwiefern diese durch Deutungsangebote wie das eines "Europäischen Sozialmodells $«$ in ausreichendem Maße vorhanden sind, bleibt eine offene Frage.

61 Ähnlich bedenklich erscheint mir der Vorschlag einer europäischen Arbeitslosenversicherung. Inwiefern eine Öffnung der Sozialkassen »quasi unter der Hand Transferleistungen innerhalb der Union ermöglichen « (Loh, Skupien 2016, S. 599) sollte, erscheint angesichts einer erstarkenden politischen Rechten ebenfalls mehr als fraglich. Wer für internationale Sozialtransfers auf Kosten der abhängig Beschäftigten keine parlamentarischen Mehrheiten organisiert, befördert - dies zeigten zuletzt die Maßnahmen zur EuroRettung - keineswegs eine Transnationalisierung der Solidargemeinschaft, sondern riskiert einen autoritären Rechtsruck. Der konzeptionelle Fokus auf potenzielle Quellen transnationaler Solidarität führt daher mit hoher Wahrscheinlichkeit in die Irre, wenn er nicht an reale Geschehnisse im Sinne des Gewünschten rückgebunden ist.

62 Pointiert ließe sich hier also auch von einem "Sozialen Westeuropa « sprechen.

63 Gindin 2015, S. 107. 
Pessimistischen Argumenten folgend, klafft im Feld europäischer Gewerkschaftspolitik also eine Lücke zwischen Anspruch und Wirklichkeit. Folglich lassen sich nun verschiedene Schlüsse ziehen, welche nicht nur von grundlegend pessimistischen Fachvertretern geteilt werden. So beklagt Schulten ${ }^{64}$ eine "substantielle Folgenlosigkeit des ssymbolischen Euro-Korporatismus«. Während auf Brüsseler Ebene zwar anhaltend Politik betrieben werde, stelle sich eine dauerhafte institutionelle Stärkung der gewerkschaftlichen Repräsentation nicht ein, sodass nationale Gewerkschaften weiterhin als "die entscheidenden Agenten transnationaler Koordinierung ${ }^{65}$ in Erscheinung treten. Angesichts dieser Heterogenität in den nationalen Rahmenbedingungen und allgemeiner organisationaler Beharrungseffekte unter den Gewerkschaften und der auch auf lange Sicht hin unwahrscheinlichen Herausbildung einer europäischen Öffentlichkeit ist, so der pessimistische Tenor, mit der Entwicklung gemeinsamer politischer Positionen im Kerngeschäft der europäischen Gewerkschaften nicht zu rechnen. (Sub-)Nationale Konstellationen sind über Jahrhunderte gewachsen und lassen sich allem Anschein nicht über den Weg einer kurzfristigen Europapolitik harmonisieren.

Gleichzeitig stellen eurooptimistische Ideen - ob in der Selbstbeschreibung ihrer Protagonisten oder der Diagnose ihrer akademischen Fürsprecher - einen notwendigen Mythos dar, der für die politische Mobilisierung eine wichtige Bedeutung hat. Als Beobachter sollten wir mit diesen Konzepten aber möglichst vorsichtig und möglicherweise auch distanziert umgehen. Denn streng genommen würde selbst die Etablierung und Umsetzung gemeinsamer Positionen im EGB als Gradmesser einer "funktionierenden " Gewerkschaftsbewegung kaum ausreichen. Der EGB könnte schließlich in allen relevanten Fragen gemeinsame Forderungen entwickeln und proklamieren und die EU sich gleichwohl zu einer noch effektiveren "Liberalisierungsmaschine «66 entwickeln. Um die realen Möglichkeiten und Grenzen europäischer Gewerkschaftspolitik abschätzen zu können, sollte daher, mit Hans-Jürgen Urban ${ }^{67}$ gesprochen, die "naive Europafreundlichkeit « einer Reihe von Fachvertretern und Praktikern "einem reflektierten Europarealismus weichen ". Solidarische Kooperation im grenzüberschreitenden Maßstab erfordert zuallererst eine möglichst realistische Einschätzung ihrer Voraussetzungen. Idealerweise hilft die Sozialwissenschaft dabei, eine solche zu ermöglichen.

\section{Literatur}

Aust, Andreas; Leitner, Sigrid; Lessenich, Stephan 2002. »Konjunktur und Krise des Europäischen Sozialmodells. Ein Beitrag zur politischen Präexplantationsdiagnostik ", in Politische Vierteliahresschrift 43,2, S. 272-301.

Bernaciak, Magdalena 2010. "Cross-border competition and trade union responses in the enlarged EU: evidence from the automotive industry in Germany and Poland «, in European Journal of Industrial Relations 16, 2, S. 119-135.

64 Schulten 2000, S. 238.

65 Schroeder, Weinert 2004.

66 Streeck 2013.

67 Urban 2009, S. 19. 
Brunkhorst, Hauke 2014 a. "Auswege aus der technokratischen Falle? Die unbeachtete Wirtschaftsverfassung Europas ", in Leviathan 42, 4, S. 508-523.

Brunkhorst, Hauke 2014 b. Das doppelte Gesicht Europas. Berlin: Suhrkamp.

Durkheim, Emile 1966. The division of labor in society. New York: The Free Press.

EGB (Europäischer Gewerkschaftsbund) 2012. Solidarity in the crisis and beyond. Discussion Note for the ETUC Winter School in Copenhagen, 7-8 February 2012.

Elias, Norbert; Scotson, John L. 2002. Etablierte und Außenseiter. Frankfurt a. M.: Suhrkamp.

Gerhards, Jürgen; Lengfeld, Holger 2013. Wir, ein europäisches Volk? Wiesbaden: VS Verlag für Sozialwissenschaften.

Gindin, Sam 2015. "Bringing class back in «, in Global Labour Journal 6. https://escarpmentpr ess.org/globallabour/article/view/2465 a (Zugriff vom 19.10.2017).

Glassner, Vera 2009. Two paths towards Europeanisation. Wien: Universität Wien.

Gumbrell-McCormick, Rebecca; Hyman, Richard 2013 Trade Unions in Western Europe. Oxford: Oxford University Press.

Haggren, Heidi; Götz, Norbert. Hrsg. 2009. Regional cooperation and international organizations. London: Routledge.

Hancké, Bob 2013. Unions, Central Banks and EMU. Oxford: Oxford University Press.

Hilson, Mary 2008. The Nordic model. Scandinavia since 1945. London: Reaction Books.

Höpner, Martin 2015. "Der integrationistische Fehlschluss", in Leviathan 43, 1, S. 29-42.

Höpner, Martin; Lutter, Mark 2014. One currency and many modes of wage formation: why the Eurozone is too heterogeneous for the Euro. MPIfG Discussion Paper 14/14. Köln: MaxPlanck-Institut für Gesellschaftsforschung.

Höpner, Martin; Schäfer, Armin. Hrsg. 2008. Die Politische Ökonomie der europäischen Integration. Frankfurt a. M., New York: Campus.

Höpner, Martin; Schäfer, Armin 2012. Integration among unequals. MPIfG Discussion Paper 12/5. Köln: Max-Planck-Institut für Gesellschaftsforschung.

Hoffmann, Jürgen 2010. "Perspektiven der europäischen Arbeitsbeziehungen und Gewerkschaften zwischen Modernisierung, Europäisierung und Globalisierung ", in Leviathan 38, 1, S. 89-102.

Hyman, Richard 2001. Understanding trade union identities. London: Sage.

Hyman, Richard 2011. "Gewerkschaftliche Strategien und Solidaritätspolitik unter globalen Konkurrenzbedingungen ", in Solidarität über Grenzen. Gewerkschaften vor neuer Strandortkonkurrenz, hrsg v. Gerlach, Frank et al., S. 51-72. Berlin: edition sigma.

IndustriALL 2012 IndustriALL European Trade Union Wage Coordination Rule. Konferenz am 27./28.11.2012 in Luxemburg.

IndustriALL 2014. »Unsere Zukunft verhandeln! « Gewerkschaftliche Strategien in Zeiten der Wirtschaftskrise. Dokument zur Tarif- und sozialpolitischen Konferenz von IndustriALL, Wien, 12.-13. Juni 2014

Kädtler, Jürgen 2013. "Tarifpolitik und tarifpolitisches System ", in Handbuch Gewerkschaften in Deutschland, hrsg. v. Schroeder, Wolfgang, S. 425-464. Wiesbaden: Springer VS.

Keune, Marten 2008. "Die Grenzen der europäischen Arbeitsmarktintegration: Koalitionen, Interessenvielfalt und institutionelle Hindernisse ", in Die Politische Ökonomie der europäischen Integration, hrsg. v. Höpner, Martin; Schäfer, Armin, S. 279-309. Frankfurt a. M., New York: Campus.

Keune, Maarten 2012. "The social dimension of European integration «, in Economy and society in Europe: a relationship in crisis, hrsg. v. Burroni, Luigi et al., S. 19-39. Cheltenham: Edward Elgar.

Kowalsky, Wolfgang 2014. "Europäischer Gewerkschaftsbund: politische Entwicklung ", in Handbuch Gewerkschaften in Deutschland, hrsg. v. Schroeder, Wolfgang, S. 617-636. Wiesbaden: Springer VS.

Krings, Torben (2015). "Bewegungsfreiheit in einem Unglcichheitsraum « in Horizontale Europäisierung im Feld der Arbeitsbeziehungen, hrsg. v. Pernicka, Susanne, S. 87-110. Wiesbaden: Springer VS.

Loh, Wulf; Skupien, Stephan 2016. "Die EU als Solidargemeinschaft ", in Leviathan 44, 4, S. 578-603.

Meardi, Guglielmo 2012. Social failures of EU enlargement. London: Routledge. 
Pernicka, Susanne 2015. "Einleitung ", in Horizontale Europäisierung im Feld der Arbeitsbeziebungen, hrsg. v. Pernicka, Susanne, S. 1-16. Wiesbaden: Springer VS.

Pierson, Paul; Leibfried Stephan 1998. "Mehrebenen-Politik und die Entwicklung des sozialen Europa" in Standort Europa. Sozialpolitik zwischen Nationalstaat und europäischer Integration, hrsg. v. Pierson, Paul; Leibfried, Stephan, S. 11-57. Frankfurt a. M.: Suhrkamp.

Platzer, Hans-Wolfgang 2010. Europäisierung der Gewerkschaften. Bonn: Friedrich-Ebert-Stiftung.

Pleines, Heiko 2008. Already arrived in Brussels? Arbeitspapiere und Materialien No. 91. Bremen: Forschungsstelle Osteuropa Bremen.

Ramsay, Harvie 1999. "In search of international union theory ", in Globalization and patterns of labour resistance, hrsg. v. Waddington, Jeremy, S. 192-239. London: Macmillan.

Schäfer, Armin; Streeck, Wolfgang 2008. "Korporatismus in der Europäischen Union ", in Die Politische Okonomie der europäischen Integration, hrsg. v. Höpner, Martin; Schäfer, Armin, S. 203-240. Frankfurt a. M., New York: Campus.

Scharpf, Fritz W. 2008. „Der einzige Weg ist, dem EuGH nicht zu folgen «, in Die Mitbestimmung $7+8$, S. $18-23$.

Scharpf, Fritz 2012. Legitimacy intermediation in the multilevel European polity and its collapse in the Euro crisis. MPIfG Discussion Paper 12/6. Köln: Max-Planck-Institut für Gesellschaftsforschung.

Schroeder, Wolfgang; Weinert, Rainer 2004. "Designing institutions in European industrial relations: a strong commission versus weak trade unions? ", in European Journal of Industrial Relations 10, 2, S. 199-217.

Schulten, Thorsten 2000. "Zwischen nationalem Wettbewerbskorporatismus und symbolischen Euro-Korporatismus", in Die Konfiguration Europas. Dimensionen einer kritischen Integrationstheorie, hrsg. v. Bieling, Hans-Jürgen; Steinhilber, Jochen, S. 222-242. Münster: Westfälisches Dampfboot.

Schulten, Thorsten 2005. "Gewerkschaften und europäische Integration", in Handlungsfeld europäische Integration, hrsg. v. Beerhorst, Joachim; Urban, Hans-Jürgen, S. 14-36. Hamburg: VSA.

Schulten, Thorsten 2013. WSI Mindestlobnbericht 2013. Düsseldorf: WSI.

Schulten, Thorsten et al. 2005. "Thesen für eine europäische Mindestlohnpolitik «, in Mindestlöbne in Europa, hrsg. v. Schulten, Thorsten et al., S. 301-306. Hamburg: VSA.

Seeliger, Martin 2017. Die soziale Konstruktion organisierter Interessen. Frankfurt a. M., New York: Campus.

Streeck, Wolfgang 1998. "The internationalization of industrial relations in Europe: prospects and problems", in Politics and Society 26, 4, S. 429-459.

Streeck, Wolfgang 2013. Gekaufte Zeit. Berlin: Suhrkamp.

Urban, Hans-Jürgen 2009. "Zeit für eine politische Neuorientierung ", in Internationale Politik und Gesellschaft. http://library.fes.de/pdf-files/ipg/ipg-2009-4/4-09_urban_d.pdf (Zugriff vom 19.10.2017).

Waddington, Jeremy; Hoffmann, Reiner 2000. Trade Unions in Europe. Brussels: ETUI.

Wiesenthal, Helmut 1992. "Das Trilemma strategisch ambitionierter Mitgliederverbände", in Berliner Journal für Soziologie 3, 1, S. 3-18.

Woll, Cornelia; Jacquot, Sophie 2010. „Using Europe: strategic action in multi-level politics «, in Comparative European Politics 8, 1, S. 110-126.

Zürn, Michael 2013. "Nachwort: Die Finanz- und Schuldenkrise", in Die Politisierung der Weltpolitik, hrsg. v. Zürn, Michael; Ecker-Erhardt, Marthias, S. 413-425. Berlin: Suhrkamp. 
Zusammenfassung: Können Gewerkschaften im Prozess der europäischen Integration eine gemeinsame politische Linie entwickeln? Anhand der Analyse gewerkschaftlicher Positionsbildung in den drei wichtigsten Feldern europäischer Tarifpolitik (die Diskussionen um die Dienstleistungsfreiheit und den europäischen Mindestlohn sowie die Lohnkoordinierung) argumentiert der Beitrag für eine ausgewogenere Sicht der realen Möglichkciten und Grenzen internationaler Solidarität.

Stichworte: europäische Gewerkschaften, EU, Tarifpolitik, internationale Solidarität

\section{Solidarity in the EU - a trade union perspective}

Summary: Can trade unions develop common political positions in the course of European integration? Based on an empirical analysis of position-making in the three most important policy fields (freedom of services, European minimum wages and wage coordination), the article argues for a more differentiated perspective on the potentials and limitations of international solidarity.

Keywords: European trade unions, European Union, collective bargaining, international solidarity

Autor

Martin Seeliger

Europa Universität Flensburg

Gebäude Helsinki, Raum 134

Auf dem Campus 1 a

24943 Flensburg

martin.secliger@gmx.net 